\title{
Determination of stress, cracks and defects density in crystals after wafer-bonding processes: a novel HRXRD - X-ray micro CT conjoint analytical approach
}

\author{
A. Borzì ${ }^{1}$, R. Zboray ${ }^{1}$, S. Dolabella ${ }^{1,2}$, A. Dommann ${ }^{1}$, A. Neels ${ }^{1,2}$ \\ ${ }^{1}$ Center for X-ray Analytics, Empa, Swiss Federal Laboratories for Materials Science and Technology, Überlandstrasse 129, CH- \\ 8600 Dübendorf, Switzerland. ${ }^{2}$ Department of Chemistry, University of Fribourg, Avenue de l'Europe 20, 1700 Fribourg, Switzerland \\ aurelio.borzi@empa.ch
}

Functional devices such as sensors, actuators or micro-electromechanical systems (MEMS) are obtained through a large variety of microfabrication processes, many of whom affect the structure and microstructure of materials because of the introduction of stress, strain, crystalline defects and volume-cracks. The materials degradation originated by these effects may translate into a lack of performance and reliability of the final devices. Indeed, in the frame of the microfabrication industry, controlling the structure of materials at the micrometer and nanometer scale represents a fundamental objective toward the optimization of the microfabrication process itself and achievement of improved devices' performance and lifetime.

In this work, we studied the influence of an innovative wafer bonding process, namely Impulse Current Bonding (ICB), in principle enabling low-temperature bonding between a wide class of materials, on the degradation of SCSi, SC-sapphire and borosilicate glass structures and crystallinity. A comprehensive frame of the microstructural deterioration at different size scales is obtained by a correlative approach between high-resolution X-ray diffraction (HRXRD)[1-2] and X-ray micro-computed tomography (CT). In particular, micro CT revealed the formation of large cracks with thickness in the order of tens of microns generated to release the high stress at the bonding interface. In parallel, strain and tilt affecting the SCSi crystallinity due to the presence of defects at the nanoscale dimension are revealed by HRXRD methods, such as the mapping of the reciprocal space (RSM), radial scans (i.e., 2 $\theta / \theta$ ) and angular scans (i.e., $\omega$-scans or rocking curves) of symmetrical and asymmetrical reflections. The residual stress after the bonding process is also calculated from the in-plane and out-of-plane X-ray strain. The effectiveness and strength of the bonding are also assessed by our approach and compared to the conventional wafer bonding technologies, i.e., the anodic bonding.

We aim to present here a unique approach to the evaluation of the structural and crystalline degradation of materials involved in wafer bonding microfabrication processes. The combination of X-ray micro CT with HRXRD enables a holistic evaluation of the bonding between SCSi, sapphire and borosilicate glass wafers achieved exploiting an innovative low-temperature process, namely ICB. This allows the correlation between the micrometer scale and volumetric defect detection (voids and cracks) with atomic-level strain and defect analysis.

(a)

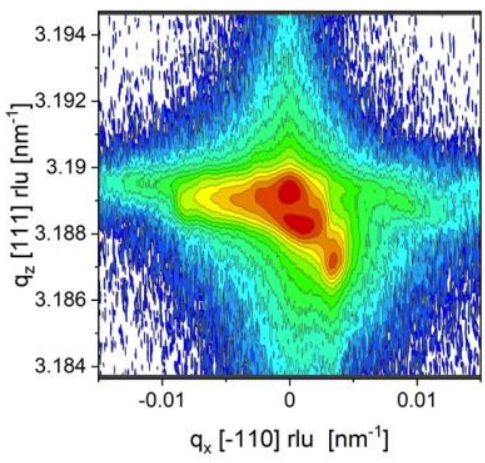

(b)

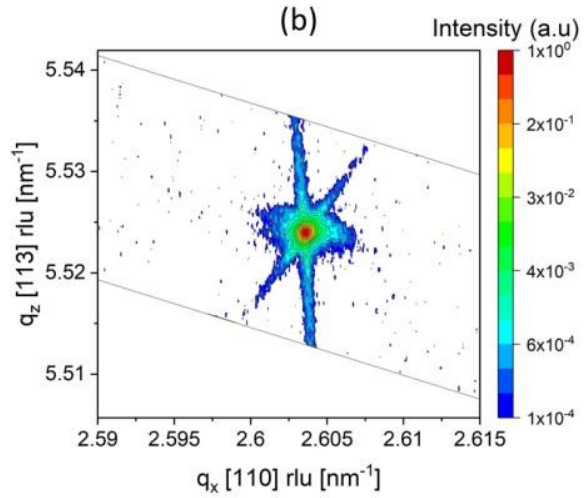

(c)

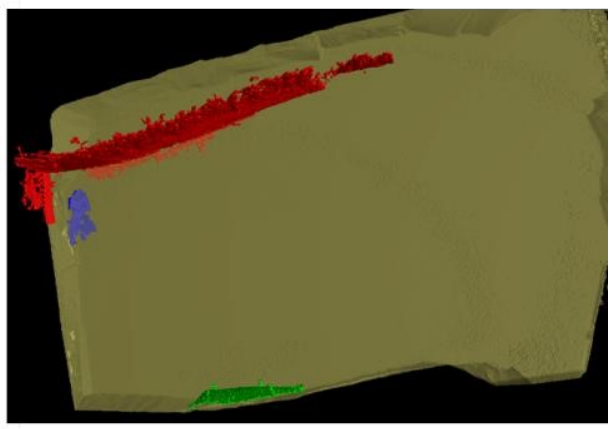

Figure 1. Distribution of the scattering vector module in the reciprocal space: (a) RSM around the symmetrical Si(111) lattice point of SCSi bonded with SC-sapphire; (b) RSM around the asymmetrical Si(113) of SCSi bonded with borosilicate glass. (c) X-ray micro CT image of cracks and voids at the bonding interface between SCSi and sapphire: Si in yellow, sapphire transparent.

[1] A. Schifferle, A. Dommann, and A. Neels, (2017) Science and Technology of Advanced Materials 18, no. 1, $219-230$.

[2] A. Neels et al., (2010), 11th international Workshop on Stress-Induced Phenomena in Metallization, 114-119

Keywords: HRXRD, X-ray micro CT, wafer bonding, microfabrication, Impulse Current Bonding (ICB) 